\title{
OTDM-to-WDM Conversion of Complex Modulation Formats by Time-Domain Optical Fourier Transformation
}

Palushani, Evarist; Richter, T.; Ludwig, R.; Schubert, C.; Mulvad, Hans Christian Hansen; Clausen, Anders; Oxenløwe, Leif Katsuo

Published in:

OFC/NFOEC Technical Digest

Publication date:

2012

Document Version

Publisher's PDF, also known as Version of record

Link back to DTU Orbit

Citation (APA):

Palushani, E., Richter, T., Ludwig, R., Schubert, C., Mulvad, H. C. H., Clausen, A., \& Oxenløwe, L. K. (2012). OTDM-to-WDM Conversion of Complex Modulation Formats by Time-Domain Optical Fourier Transformation. In OFC/NFOEC Technical Digest [6191950] Optical Society of America.

\section{General rights}

Copyright and moral rights for the publications made accessible in the public portal are retained by the authors and/or other copyright owners and it is a condition of accessing publications that users recognise and abide by the legal requirements associated with these rights.

- Users may download and print one copy of any publication from the public portal for the purpose of private study or research.

- You may not further distribute the material or use it for any profit-making activity or commercial gain

- You may freely distribute the URL identifying the publication in the public portal 


\title{
OTDM-to-WDM Conversion of Complex Modulation Formats by Time-Domain Optical Fourier Transformation
}

\author{
E. Palushani ${ }^{1}$, T. Richter ${ }^{2}$, R. Ludwig ${ }^{2}$, C. Schubert ${ }^{2}$, H. C. H. Mulvad ${ }^{1}$, A. T. Clausen ${ }^{1}$ and L. K. Oxenløwe ${ }^{1}$ \\ ${ }^{1}$ DTU Fotonik, Technical University of Denmark, Øresteds Plads 343, 2800 Kgs. Lyngby, Denmark \\ ${ }^{2}$ Fraunhofer Institute for Telecommunication, Heinrich-Hertz-Institut, Einsteinufer 37, 10587 Berlin, Germany \\ epalu@fotonik.dtu.dk
}

\begin{abstract}
We demonstrate the utilization of the optical Fourier transform technique for serial-toparallel conversion of $64 \times 10-G B d$ OTDM data tributaries with complex modulation formats into $50-\mathrm{GHz}$ DWDM grid without loss of phase and amplitude information.

OCIS codes: (070.4340) Nonlinear optical signal processing; (320.7160) Ultrafast Technology, (060.1660) Coherent communications
\end{abstract}

\section{Introduction}

Serial-to-parallel conversion is one of the functionalities that might be necessary in future photonic networks, in which ultra-fast optical signals of any bit rate and modulation format will be transmitted and processed from end-toend in the optical domain. Traditionally, optical time division multiplexing (OTDM) has been utilized to push the serial data rate on a single wavelength far beyond electronic speeds. In combination with complex modulation formats (e.g. 16-QAM) and polarization multiplexing, up to $10.2 \mathrm{Tbit} / \mathrm{s}$ has been realized [1]. At such high speeds, the complexity of the receiver scales with the number of OTDM tributaries. A serial-to-parallel conversion would take advantage of the mature technology used in wavelength division multiplexing (WDM) systems for parallel channel extraction.

In this paper, we demonstrate the utilization of the optical Fourier transform (OFT) technique for single step [2] conversion of several 10-GBd tributaries of a 64x10-GBd OTDM signal encoded with complex modulation formats onto a $50-\mathrm{GHz}(0.4 \mathrm{~nm})$ dense WDM (DWDM) grid. The data signal is generated with return-to-zero (RZ) quadratue phase-shift keying (QPSK) modulation and RZ 16-ary quadrature-amplitude modulation (16-QAM). The OFT is performed via dispersion followed by phase modulation using linearly chirped pump pulses in a four-wave mixing (FWM) process [2]. The time-to-frequency conversion results in spectral compression [3] which maps the different OTDM tributaries into separate DWDM channels and preserves the phase and amplitude information encoded on them. The resulting 10-GBd DWDM channels are demodulated in a coherent heterodyne receiver using a continuous-wave $(\mathrm{CW})$ laser as local oscillator (LO). The resulting DWDM channels are found to be error-free assuming $7 \%$ hard decision FEC.

\section{Principle of Operation and Experimental Setup}

Time-to-frequency mapping via OFT is achieved by propagation of the waveform to be converted (OTDM tributaries in this case) in a dispersive medium with second order chromatic dispersion $D$, followed by quadratic phase modulation with chirp rate $C$ [2-4]. The quadratic phase modulation is achieved in a FWM process with linearly-chirped pump pulses $[2,3]$. If the condition $D=1 / C$ is fulfilled, the OFT technique transfers the time information into the frequency domain. In case of FWM, the pump chirp and data chirp are combined in the idler signal where they cancel each other, hence converting the OTDM tributaries to spectrally compressed DWDM channels. The time spacing $(\Delta \tau)$ in the original signal and the angular frequency spacing $(\Delta \omega)$ in the power spectrum of the Fourier-transformed one (FWM idler in this case) are related by the simple equation: $\Delta \omega=C \cdot \Delta \tau$. The $D$ and $C$ parameters can be used to control the amount of spectral compression that can be achieved at the transformed signal. Here, the parameters are chosen such that a $640-\mathrm{GBd}$ tributary spacing of $\Delta \tau=1.56 \mathrm{ps}$ is mapped to a

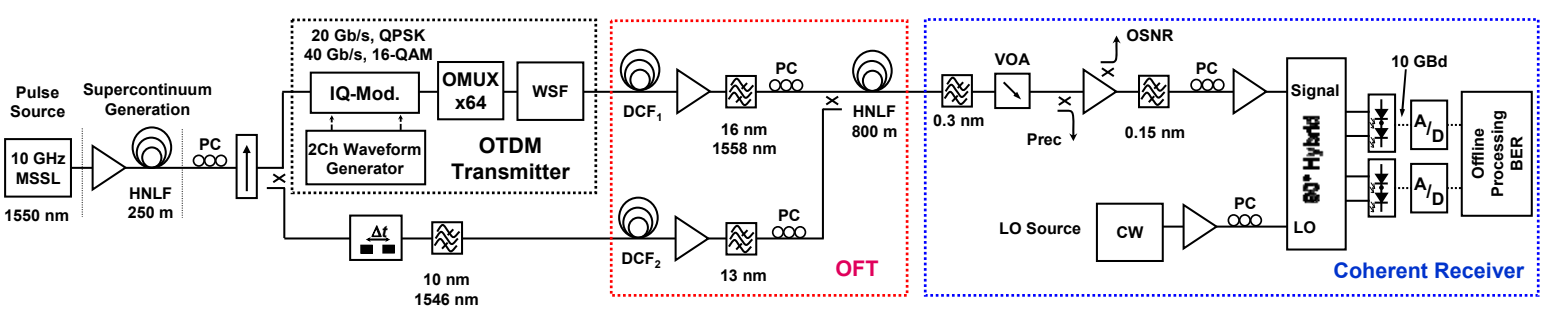

Fig. 1: Schematic depiction of the experimental setup. 
DWDM spacing of $\Delta \omega /(2 \pi)=50 \mathrm{GHz}(0.4 \mathrm{~nm})$.

Fig. 1 shows the experimental setup. It is composed of the OTDM transmitter, the OFT block for serial-toparallel conversion and the coherent receiver. At the transmitter side, a mode-locked solid state laser (MSSL) produces a train of pulses having 1.2-ps full-width at half-maximum (FWHM), 10-GHz (STM-64) repetition rate and center wavelength at $1550 \mathrm{~nm}$. The pulses are amplified to $21.5 \mathrm{dBm}$ and injected into a $250-\mathrm{m}$ long highlynonlinear fiber (HNLF) with attenuation $\alpha=0.78 \mathrm{~dB} / \mathrm{km}, \quad$ dispersion $D=-0.49 \mathrm{ps} /(\mathrm{nm} \cdot \mathrm{km})$, slope $S=0.0061 \mathrm{ps} /\left(\mathrm{nm}^{2} \cdot \mathrm{km}\right)$ at $1550 \mathrm{~nm}$ and nonlinear coefficient $\gamma=10(\mathrm{~W} \cdot \mathrm{km})^{-1}$. The optical spectrum at the output of the HNLF is broadened due to self-phase modulation (SPM), resulting in a wide supercontinuum (SC) exceeding $40 \mathrm{~nm}$. The broad spectrum is used to generate both data and pump for the FWM process. The pump spectrum is carved out of the SC by a 10-nm wide flat-top band-pass filter (BPF) centered at $1546 \mathrm{~nm}$. To obtain data the SC is injected in an in-phase and quadrature modulator (IQ-Mod.). The IQ modulator is driven by a two-channel arbitrary waveform generator which is programmed to generate QPSK or 16-QAM modulation (De Bruijn sequence of $2^{15}$ before symbol mapping) resulting in respectively a $20-$ or $40-\mathrm{Gbit} / \mathrm{s}$ data signal. This signal is time interleaved up to 640 GBd in a fiber-based delay line multiplexer (OMUX). At the output of the OMUX stage, the SC is filtered by a wave-shaping filter (WSF), liquid crystal on silicon (LCoS) technology, in order to generate Gaussian-like pulses of 600 -fs duration centered at $1558 \mathrm{~nm}$. The signal is then launched into the OFT block.

The data path of the OFT block consists of dispersion compensating fiber $\left(\mathrm{DCF}_{1}\right.$ in Fig. 1$)$ used to compensate 244-m single mode fiber (SMF). DCF $_{2}$ (Fig. 1), equivalent to 501-m SMF, is used to linearly chirp the pump pulses. Fine tuning of the total data dispersion, in order to comply with the OFT requirements, is achieved by the phase shaping ability of the WSF. Once dispersed, data and pump are injected into an 800-m HNLF for the FWM process. The used HNLF has $D=0.18 \mathrm{ps} /(\mathrm{nm} \cdot \mathrm{km}), S=0.018 \mathrm{ps} /\left(\mathrm{nm}^{2} \cdot \mathrm{km}\right)$ at $1550 \mathrm{~nm}$ and $\gamma=10(\mathrm{~W} \cdot \mathrm{km})^{-1}$. At the output of the HNLF a tunable 0.3-nm BPF is used to select the desired DWDM channel from the FWM idler. This 10-GBd channel is sent to the coherent receiver for demodulation and BER characterization.

The data path of the pre-amplified receiver consists of a variable optical attenuator (VOA) to vary the received signal power and OSNR, two erbium-doped fiber amplifiers (EDFA), a 90 ${ }^{\circ}$-hybrid, two balanced photo-detectors (BPD), two analog-to-digital converters (A/D, 50-GS/s real-time sampling oscilloscope with $20 \mathrm{GHz}$ bandwidth) and a computer for offline processing and BER measurement. A tunable CW laser is used as LO. The optical input powers into the $90^{\circ}$-hybrid are $12 \mathrm{dBm}$ for the data signal and $14 \mathrm{dBm}$ for the $\mathrm{LO}$. The data is recovered by a conventional digital signal processing block comprising resampling, relative carrier frequency offset compensation (data signal with respect to LO) and blind adaptive equalization. After pre-convergence of the equalizer using the constant modulus algorithm, it was switched to a decision-directed least-mean square filter error criterion.

\section{Results and Discussion}

Fig. 2 a) and b) show the spectra at the output of the HNLF in case of 320- and 640-GBd RZ-QPSK OTDM data signals. A zoom-in on the idler spectrum is shown in Fig. 2 c). More than 17 tributaries are time-to-frequency mapped in case of 320-GBd OTDM signal, with a grid spacing of $100 \mathrm{GHz}$, and more than 35 tributaries are mapped in case of $640 \mathrm{GBd}$, with a grid spacing of $50 \mathrm{GHz}$ (range 1527.5-1540 nm).

Fig. 3 a) shows the BER vs. OSNR curve of the 10-GBd converted tributary centered at $1533.5 \mathrm{~nm}$. The performance is compared with three back-to-back (B2B) curves measured for a 10-GBd RZ and a 10-GBd nonreturn to zero (NRZ) QPSK signal centered at the idler and data-signal wavelengths. The three B2B curves were measured in order to confirm the wavelength independence of the receiver and the modulation quality of the RZ pulses. As it can be seen, there is negligible penalty in case of 160- and 320-GBd OTDM signals. However, due to the HNLF length, the performance of the system is worse at $640-\mathrm{GBd}$. This is related to inter-symbol interference caused by the walk-off between pump and data. To verify the integrity of the entire OTDM signal, each tributary is
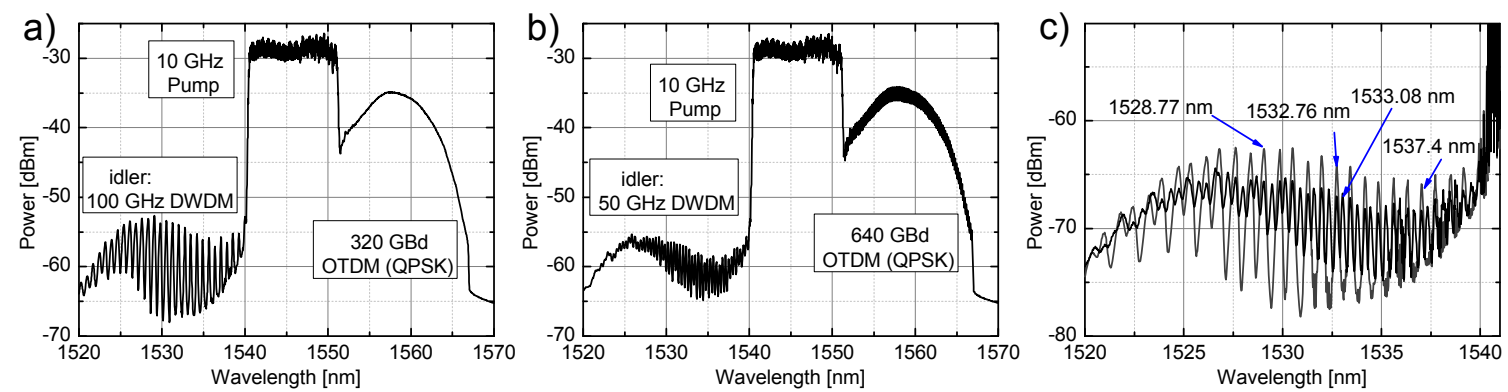

Fig. 2: a) and b) spectra at HNLF output in case of 320- and 640-GBd RZ-QPSK. c) Zoom-in on the DWDM idler for both 320 and 640 GBd. 

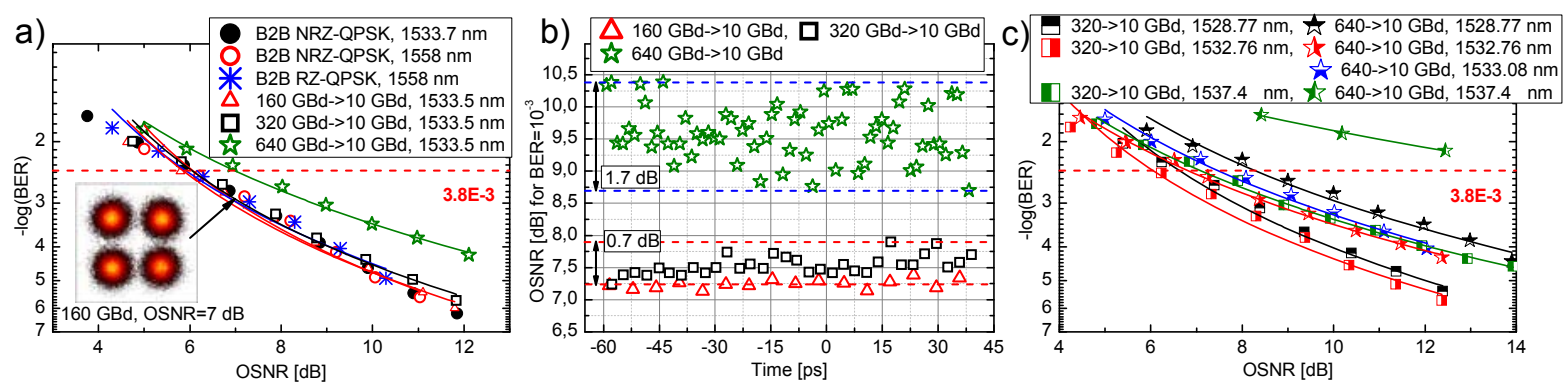

Fig. 3: a) BER curves for one converted 10-GBd tributary at $1533.5 \mathrm{~nm}$ in case of 160-, 320- and 640-GBd RZ-QPSK OTDM. Inset: Constellation for one 10-GBd channel out of a converted 160-GBd OTDM QPSK signal at 7-dB received OSNR $\left(\mathrm{BER}=10^{-3}\right)$. b) Time scan of all tributaries with required OSNR for $B E R=10^{-3} \mathrm{c}$ ) BER performance for various DWDM channels.

extracted by keeping the $0.3 \mathrm{~nm}$ BPF fixed to $1533.5 \mathrm{~nm}$ and by changing the time delay $\Delta t$ (Fig. 1) between pump and OTDM tributaries. This results in a frequency scan of the tributaries at the FWM idler. Fig. $3 \mathrm{~b}$ ) shows the measured OSNR in order to reach a BER $=10^{-3}$ for all the OTDM tributaries in case of 160-, 320- and 640-GBd RZQPSK OTDM. Fig. $3 \mathrm{c}$ ) shows the BER performance for different DWDM channels measured by keeping $\Delta t$ fixed and by tuning the $0.3 \mathrm{~nm}$ BPF and the wavelength of the LO. Compared to the central DWDM channels, the ones closer to the pump have a worse BER performance due to interference with residual pump light.

Finally the OFT technique is tested also on 16-QAM. Fig. 4 shows the BER curve for the converted 10-GBd tributary centered at $1530.4 \mathrm{~nm}$ in case of 160- and 320-GBd RZ 16-QAM OTDM. The curves are compared to the

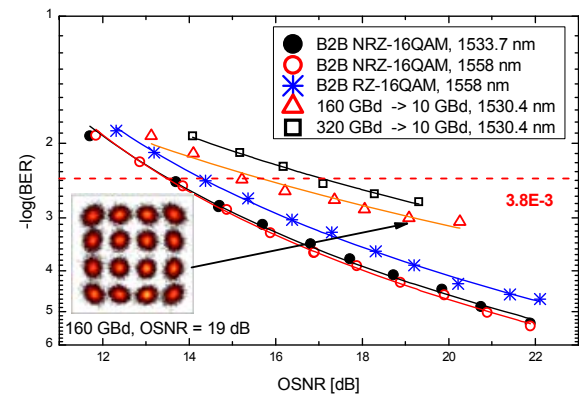

Fig. 4: BER curves for the converted 10-GBd tributary at $1530.4 \mathrm{~nm}$ in case of 160- and 320-GBd RZ 16-QAM OTDM. Inset: Constellation for one 10-GBd channel out of a converted 160-GBd OTDM QPSK signal at 19-dB received OSNR $\left(B E R=10^{-3}\right)$.

B2B cases for 10-GBd RZ and NRZ 16-QAM (at data and idler wavelengths). Both BER curves are below the $3.8 \times 10^{-3}$ threshold value for $7 \%$ overhead FEC. This confirms the fact that the OFT technique preserves the phase and amplitude information encoded on the converted OTDM tributaries. At $640 \mathrm{GBd}$ the system did not comply with the FEC specifications. This is related to the walk-off between pump and data signal. An improved performance is expected in case of an HNLF without walk-off.

\section{Conclusion}

We demonstrated the utilization of a FWM-based time-domain OFT for serial (OTDM) to parallel (DWDM) conversion of advanced modulation formats. This technique was successfully demonstrated for 160- to 640-GBd RZ-QPSK and 160- to 320-GBd RZ 16-QAM. It was shown that the serial-to-parallel conversion process preserves the modulation format of the OTDM tributaries.

\section{Acknowledgement}

This work was sponsored by the Danish Research Council within the NOSFERATU project and partly by the German Federal Ministry of Education and Research with the program Optical Technologies (contract 13N9356).

\section{References}

[1] T. Richter, E. Palushani, C. Schmidt-Langhorst, M. Nölle, R. Ludwig, J.K. Fischer, and C. Schubert, "Single wavelength channel 10.2 Tb/s TDM-data capacity using 16-QAM and coherent detection", Optical Fiber Communication Conference, OFC 2011, paper PDPA9, (2011).

[2] H. C. H. Mulvad, E. Palushani, M. Galili, J. Xu, H. Hu, A. Clausen, L. Oxenløwe, and P. Jeppesen, "OTDM-WDM conversion based on timedomain optical Fourier transformation with spectral compression”, Optical Fiber Communication Conference, OFC 2011, paper OThN2, (2011).

[3]. E. Palushani, H.C.H. Mulvad, M. Galili, H. Hu, L.K. Oxenløwe, A. Clausen, and P. Jeppesen, "OTDM-to-WDM Conversion Based on Timeto-Frequency Mapping by Time-Domain Optical Fourier Transformation”, IEEE JSTQE, DOI: 10.1109/JSTQE.2011.2131121, in print (2011).

[4] T. Hirooka, M. Nakazawa, "Optical Adaptive Equalization of High-Speed Signals Using Time-Domain Optical Fourier Transformation", J.Lightw. Technol., 24, 7, 2530-2540, July 2006. 